\title{
Probabilistic approach to the analysis of regularities in behavior of material parameters of electronic equipment under action of external fields
}

\author{
G.V. Milenin \\ V. Lashkaryov Institute of Semiconductor Physics, National Academy of Sciences of Ukraine, \\ 41, prospect Nauky, 03028 Kyiv, Ukraine
}

\begin{abstract}
It has been shown that the dependence between the parameters of materials of electronic equipment and external fields is determined by the distribution function of the corresponding random variable. The obtained results have been applied to the analysis of a number of physical phenomena.
\end{abstract}

Keywords: random variable, distribution function, defect generation, spin-dependent reaction, magnetization of ferromagnetics.

Manuscript received 08.04.15; revised version received 04.08.15; accepted for publication 28.10.15; published online 03.12.15.

\section{Introduction}

As it was noted in [1], the effects of electric, magnetic, electromagnetic and radiation fields on materials of semiconductor products lead to changes in states of ensembles of particles and defects. Behavior of these ensembles is caused by flow of random events in physical and chemical processes. This concept allowed to give mathematical description of proceeding with time a number of physical and chemical processes from the probability positions. Therefore, it is of interest to develop a probabilistic approach to the analysis of the dependence of parameters of electronic equipment material on the values of the parameters characterizing the external fields (hereinafter, in the broadest sense, we will call these parameters as the force ones).

\section{Statistic regularities of behavior inherent to material parameters under action of external fields}

Let the probability that a particle, defect or structural formation in material does not change its state when the parameter of the external field changes from 0 to $f$ will be $P(f)$. Furthermore, changes in a state, position, generation or annihilation of particle, defect and structural formation under external influences will be treated as a random event (or simply - event). Then the probability of the lack of events when changing the force parameter to $f+d f$ can be presented in the form $P(f+d f)$. The latter probability can be considered as the probability of a complex event consisting in the absence of the event when changing the force parameter from 0 to $f$ and in the range $d f$. Let us assume that these events are independent. When these events are independent, the sought-for probability is equal to the product of the probabilities of the component events:

$$
P(f+d f)=P(f) P(d f) .
$$

Let $\beta(f) d f$ is the probability of event in the range $d f$. Then the probability of the opposite event is equal to $1-\beta(f) d f$.

Consequently,

$$
P(f+d f)=P(f)[1-\beta(f) d f],
$$

and $\beta(f)$ will be called as the sensitivity of events to the parameters of external fields (hereinafter - the sensitivity of events). The mathematical definition of the latter will be given below. 
Considering the mathematical definition of a derivative, we obtain

$$
P(f+d f)=P(f)+\frac{d P(f)}{d f} d f .
$$

Substituting (3) into (2), we get

$$
d P(f)=-\beta(f) P(f) d f .
$$

Integrating (4), we find

$$
P(f)=e^{-\int \beta(f) d f} \text {. }
$$

Then for the probability of event to the value of parameter of the external field $f$, we get

$$
F(f)=1-e^{-\int_{0}^{f} \beta(f) d f} .
$$

In other words, $F(f)$ is the distribution function of the random variable - the force parameter before the event.

As it is seen from (5) and (6), the sensitivity of events is determined as follows:

$\beta(f)=\frac{1}{P(f)} \frac{d F(f)}{d f}$,

where $d F(f) / d f$ - density of distribution of the force parameter before the event (the probability density of the event).

By analogy with [1], where the term of event intensity is used, we can write an expression for the sensitivity of events:

$$
\beta(f)=\frac{\Delta n(f)}{\left[n_{0}-n(f)\right] \Delta f},
$$

where $n_{0}$ is the total number of events, $n(f)$ - number of events to the force parameter value $f, \Delta n(f) / \Delta f$ number of events when changing the parameter of the external field by $\Delta f$ (the number of events per unit $f$ ).

Let us define the quantities in the formula (8) by using an example of some physical phenomena.

1. Generation of defects in semiconductor materials under the influence, for example, of laser irradiation. A random event - generation of a defect: $\beta(f)$ - the sensitivity of generations of defects to the intensity of laser irradiation $f=I ; n_{0}$ - total number of generated defects; $n(f)$ - number of defects generated to the value of the laser irradiation intensity $f=I ; \Delta n(f) / \Delta f$ - number of the defects generated per unit of the laser irradiation intensity $f=I$.

2. The spin-dependent reactions. A random event is a change in the electron state caused by a change in spin orientation: $\beta(f)$ - sensitivity of changes in electron states to the magnetic induction $f=B ; n_{0}-$ total number of electrons changing their state; $n(f)$ - number of electrons that have changed their state to the magnetic induction value $f=B ; \Delta n(f) / \Delta f$ number of electrons changed their state by the unit of magnetic induction $f=B$.

3. Magnetization of ferromagnetics. A random event is the domain wall displacement: $\beta(f)$ - sensitivity of displacements of the domain walls to the magnetic field strength $f=H ; n_{0}$ - total number of the displacing domain walls; $n(f)$ - number of domain walls, displaced to the value of the magnetic field strength $f=H ; \Delta n(f) / \Delta f$ - number of domain walls that have displaced per the unit of magnetic field strength $f=H$.

Since, by definition $F(f)=n(f) / n_{0}$, accounting (6), we get

$n(f)=n_{0}\left[1-e^{-\int^{f} \beta(f) d f}\right]$.

From (9) it follows that at $f \rightarrow \infty, n(f)$ asymptotically approaches the value $n_{0}$.

For $f$ so small that $\int_{0}^{f} \beta(f) d f<<1$, expanding the exponent in series and limiting only by the first term of the expansion, we obtain

$n(f)=n_{0} \int_{0}^{f} \beta(f) d f$.

In its turn, the changes in the parameter values of electronic equipment materials control the changes in $n(f)$ and are proportional to $n(f)$. Considering that in the initial state the material parameter had a value $y_{i n}$, then during its growth with the $f$ increase, taking (9) into account, we get

$y(f)=y_{i n}+y_{0}\left[1-e^{-\int_{0}^{f} \beta(f) d f}\right]$,

where $y_{0}$ is the asymptotically attainable value $y(f)-y_{\text {in }}$ when $f \rightarrow \infty$.

If $y(f)$ decreases with increasing $f$, we obtain

$y(f)=y_{i n}-y_{0}\left[1-e^{-\int_{0}^{f} \beta(f) d f}\right]$,

where $y_{0}$ is the asymptotically attainable value $y_{\text {in }}-y(f)$ when $f \rightarrow \infty$.

For $f$ so small that $\int_{0}^{f} \beta(f) d f<<1$, according to (10) we have 


$$
\begin{aligned}
& y(f)=y_{\text {in }}+y_{0} \int_{0}^{f} \beta(f) d f, \\
& y(f)=y_{\text {in }}-y_{0} \int_{0}^{f} \beta(f) d f .
\end{aligned}
$$

Therefore, the change in the material parameters is determined by the form of the $\beta(f)$ function that depends on the type of the used distribution $F(f)$. To analyze the force dependences of the change of material parameters, as in the case of the mathematical description of the flowing physical and chemical processes with time [1], we use the distribution of Weibull-Gnedenko, the function of which takes the form [2]:

$$
F(f)=1-e^{-\left(\frac{f}{f^{*}}\right)^{m}},
$$

where $f^{*}$ and $m$ are the distribution parameters.

The expression for $\beta(f)$, in accordance with (7) and (15), is as follows:

$\beta(f)=\frac{m}{\left(f^{*}\right)^{m}} f^{m-1}$.

In future, we will call $m$ as the form factor of distribution of a random variable - force parameter before the event, and we mean that $f^{*}$ (that is the scale parameter) is the constant of the force parameter. When $f=f^{*}$, the probability of a random event is $1-e^{-1}$, that is, about $63 \%$ of the events. When $m=1$, the sensitivity of the events is a constant, and when $m \neq 1$ it changes with increasing the parameter of the external field by a power law.

We note three different types of $F(f)$ behavior over the whole range $f$. When $0<m<1$, the curve $F(f)$ is always convex and tends asymptotically to unity. At $m>1$, the curve in the initial section of change in $f$ is concave, and then after passing through an inflection point it becomes convex, tending to the asymptote. When $m=1$, the Weibull-Gnedenko distribution transforms into the exponential law that is characterized by a linear behavior of $F(f)$ in the initial section of change in $f$.

The Weibull-Gnedenko distribution has the following important feature [3]. At $m>1$, when $3<m<4$, the Weibull-Gnedenko distribution reduces to the normal one. The function of the Weibull-Gnedenko distribution is symmetrical at the point $F(f)=0.5$ at $m=3.26$, that is $(1-\ln 2)^{-1}$, and the inflection point changes with $m$ remarkably slower. When $0<m<0.7$, the Weibull-Gnedenko distribution is reduced to the normal logarithmic one. Finally, as it was already noted, when $m=1$ the Weibull-Gnedenko distribution transforms into the exponential one.

Replacing $\beta(f)$ in the formulas (9) and (10) to (16) and integrating, we obtain $n(f)=n_{0}\left[1-e^{-\left(\frac{f}{f^{*}}\right)^{m}}\right]$,

$n(f)=n_{0}\left(\frac{f}{f^{*}}\right)^{m}$

Accordingly, substituting (16) into (11) and (12), we will get the following force dependences of changes in the material parameters:

$y(f)=y_{\text {in }}+y_{0}\left[1-e^{-\left(\frac{f}{f^{*}}\right)^{m}}\right]$,

$y(f)=y_{i n}-y_{0}\left[1-e^{-\left(\frac{f}{f^{*}}\right)^{m}}\right]$.

At the values of force parameters so small that $\left(f / f^{*}\right)^{m}<<1$, as a result of expansion of the exponent in series and limitation by the first term of the expansion, these dependences can be represented as:

$y(f)=y_{\text {in }}+y_{0}\left(\frac{f}{f^{*}}\right)^{m}$,
$y(f)=y_{\text {in }}-y_{0}\left(\frac{f}{f^{*}}\right)^{m}$.

Thus, in the case of using the Weibull-Gnedenko distribution, the change in material parameters for small $f$ is described by the power functions. On the other hand, taking into account that in practice for small $f$, the changes in the respective parameters are well approximated by power laws, it once again confirms the correctness of using the Weibull-Gnedenko distribution for mathematical analysis of random events in the materials of electronic equipment under the influence of external fields.

In the presence of the sensitivity threshold $f_{0}$ to the action of external fields, the expressions (17)-(22) can be rearranged via replacing $f$ by $f-f_{0}$.

\section{Some applications of the obtained results}

The relation (19) well approximates the dependence of the relative conductivity of $\mathrm{InSb}$ on the laser irradiation intensity, which is presented in [4] (Fig. 1, curve 1). This dependence is rectified in the Weibull-Gnedenko coordinates:

$$
z=\ln \ln \left[1-\frac{(\Delta I / I)-(\Delta I / I)_{i n}}{(\Delta I / I)_{0}}\right]^{-1} \text { and } x=\ln (I / I)_{t h}
$$

(Fig. 1, curve 2), in this case $m \approx 3.2$, that is the sensitivity of generations of defects is a power function of the laser irradiation intensity. 
The formula (19) with the form factor $m=1$ describes given in $[5,6]$ regularities of the behavior of the microhardness of crystals $\mathrm{NaCl}$ with paramagnetic impurities $\mathrm{Ca}$ and the photocurrent in fullerene and tetracene crystals depending on magnetic induction caused by spin-dependent reactions. In these cases, there is a constant sensitivity of changes in the states of electrons in the defects to the magnetic induction.

The obtained results can be recommended to use for describing the magnetization curve of ferromagnetics with the polydomain structure. The magnetization of such ferromagnetics is caused by the process of displacements of domain boundaries (domain walls) and growth of volumes of those domains, in which the magnetic moment vector forms the smallest angle with the magnetic field direction [7]. With increasing magnetic field strength, the state of material reaches the technical saturation. If we continue to strengthen the field, then there observed is the paraprocess comprising weak linear increase of magnetization [7].

Thus, the dependence $B$ on $H$ can be expressed as $B(H)=B_{1}(H)+B_{2}(H)$ : where $B_{1}(H)$ describes the process related with motion of the domain walls, which obeys the laws of probability and is described by the expression (19) with $f=H, y(f)=B(H), y_{i n}=0$ (previously demagnetized material). In its turn $B_{2}(H)=\mu \mu_{0} H$ is the paraprocess component [8], here $\mu_{0}$ is the magnetic constant, $\mu$ is the magnetic permeability for the paraprocess.

In particular, the curve of magnetization of gray cast iron is presented in the work [9]. The analysis shows that it is approximated by the expression (19) with $m \approx 1.7$. It indicates that the sensitivity to displacements of the domain walls is a power function of the magnetic field.

Similarly, one can analyze the dependences of polarizability of ferroelectrics with a polydomain structure on the electric field. Polarizability of ferroelectrics $P(E)=P_{1}(E)+P_{2}(E)$ consists of the nonlinear orientational polarizability $P_{1}(E)$ caused by the process of repolarization of domains possessing a statistical character $[7,10]$.

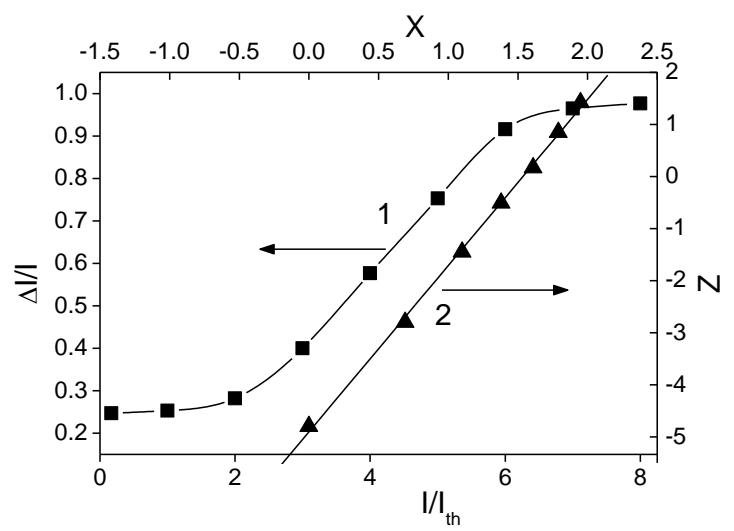

Fig. 1. Relative change of the InSb sample conductance as a function of the laser irradiation intensity.
It can be represented in the form (19) with $f=E$, $y(f)=P(E), y_{i n}=0$ as well as the contribution of the mechanisms of elastic and relaxation polarization $P_{2}(E)=$ $\varepsilon_{0} \chi E[10]$ ( $\varepsilon_{0}$ is the dielectric constant of vacuum, $\chi$ is the dielectric susceptibility by induced field of elastic and relaxation polarization) causing a weak linear increase in the segment of domain polarization saturation.

\section{Conclusions}

Behavior of material parameters of electronic equipment under the influence of external fields obeys the laws of statistics. Analytical expressions establishing the relation between the parameters of materials and external fields are the distribution functions of the corresponding random variables. The use of the Weibull-Gnedenko distribution for this purpose has been substantiated. A number of applications of the obtained results has been demonstrated.

\section{References}

1. G.V. Milenin, Analysis of random events in the physical and chemical processes flowing in materials of semiconductor products under external influences and thermal aging // Semiconductor Physics, Quantum Electronics \& Optoelectronics, 18(3), p. 233-247 (2015).

2. V.S. Koroliuk, N.I. Portenko, A.V. Skorokhod, A.F. Turbin, Reference Book on the Theory of Probabilities and Mathematical Statistics. Nauka, Moscow, 1985 (in Russian).

3. M. Brown, D. Dollimor, A. Galway, Reactions of Solids. Mir, Moscow, 1983.

4. A. Medvid', L.L. Fedorenko, V. Snitka, The mechanism of generation of donor centers in pInSb by laser radiation // Appl. Surf. Sci. 142, p. 280-285 (1999).

5. R.B. Morgunov, Spin micromechanics in the physics of plasticity // Usp. Fiz. Nauk, 174, № 2, p. 131-153 (2004).

6. Yu.I. Golovin, Magnetoplasticity of Solids (Review) // Fizika tverd. tela, 46(5), p. 769-803 (2004), in Russian.

7. V.S. Sorokin, B.P. Antipov, N.P. Lazareva, Materials and Components of Electronic Equipment (2 Volums). Vol. 2. Active Dielectrics, Magnetic Materials, Elements of Electronic Equipment. Publ. Center "Academy", Moscow, 2006 (in Russian).

8. E.G. Kalashnikov, Electricity. Nauka, Moscow, 1977 (in Russian).

9. H. Kuchling, Physik. Mir, Moscow, 1982 (in Russian).

10. Yu.M. Poplavko, L.P. Pereverzev, I.P. Raievski, Physics of Active Dielectrics. Publ. House of the Southern Federal County, Rostov/Don, 2009 (in Russian). 